\title{
Epidermophyton floccosum
}

National Cancer Institute

\section{Source}

National Cancer Institute. Epidermophyton floccosum. NCI Thesaurus. Code C127707.

A species of dermatophylic and anthropophilic fungi in the phylum Ascomycota. E.

floccosum is the causative agent of various cutaneous mycoses, but is restricted to the cornified layers of the epidermis. 